\title{
A Framework for Distributed Processing of Air Pollution Dispersion Models
}

\author{
Gerhard Eschelbeck, Christian Hartl \\ Research-Institute for Microprocessor Applications (FIM) \\ University Linz, A-4040 Linz, Austria \\ Email: eschelbeck@fim.uni-linz.ac.at
}

\begin{abstract}
The increasing pollution of our atmosphere due to organic and inorganic emissions from industry and a lot of factories require the availability of reliable but simple tools for the calculation of air pollutant emissions. Many different calculation and simulation models are approved nowadays. One difficulty is the increasing requirement of computational power. Our idea was to implement a general architecture for parallel graphical pollution simulation providing the integration of new calculation models in a very fast way. We chose an object oriented approach, which allows to integrate further calculation models and special user interface components in an efficient way. Thereby, the user can simulate environmental situations due to various air pollution dispersion models without changing the application, but by selecting the corresponding calculation model. The benefit of our solution is the separation of the input unit from the calculation and from the output unit. For reducing the system requirements on Windows / Windows NT environments, the calculation unit is based on a distributed client / server architecture. It deals with dynamic job distribution within networks for parallel computation without the need of special workstations. Thereby free resources are used for increasing performance. Standardized remote procedure calls are the basic mechanism for distributing jobs to remote computers. For analysing and benchmarking our framework we have implemented a calculation module for the approved standard OENORM M9440.
\end{abstract}

Keywords

Pollutant, Dispersion, Parallel Simulation Model, Client/Server, Distributed Calculation

\section{INTRODUCTION}

It is not our competence to discuss the amount of dispersion which is harmful or not for the mankind, that's the medical and toxicological experts' job. We will show how to process data and information of concentrations and distributions, which support government for their 
decisions. Due to low resource requirements our application opens for a large group of potential users. It gives the possibility to simulate and visualize various situations for future industry settlement planning and licensing of emittants. Furthermore it can be shown how improvements like integration of filters reflect on the emission volume.

Our application consists of an essential main component and a lot of different optional calculation modules which can be linked to the base calculation unit as add-on parts. The overall system bases on an object oriented architecture fulfilling modelling and simulation of different environmental locations under varying situations. To improve performance for simulation the base calculation unit is responsible for job distribution to remote computers depending on available resources. Partitioning of the model into subjobs must be done by the corresponding calculation module itself. The application allows to define situations with individual sources and receptor points modelling the particular environment. Receptor points are the geographical points indicated by the spatial coordinates $\mathrm{x}, \mathrm{y}$ and $\mathrm{z}$, for which the concentration of air pollutant should be determined. Sources are places where the exhausted gas - a mix of various substances - is emitted from plants into the atmosphere. We have integrated an interface for loading predefined information about local environment and geographical data (cities, mountains, streams, ...) from external files. This allows to setup background information for better preparation of outputs.

\section{A GENERAL FRAMEWORK FOR PARALLEL SIMULATION AND VISUALISATION OF ENVIRONMENTAL INFORMATION}

As mentioned above and given in figure 1 our architecture is divided into mainly three components (input, calculation and output unit). The calculation unit is subdivided into the mandatory base unit and optional calculation modules specifying various simulation models.

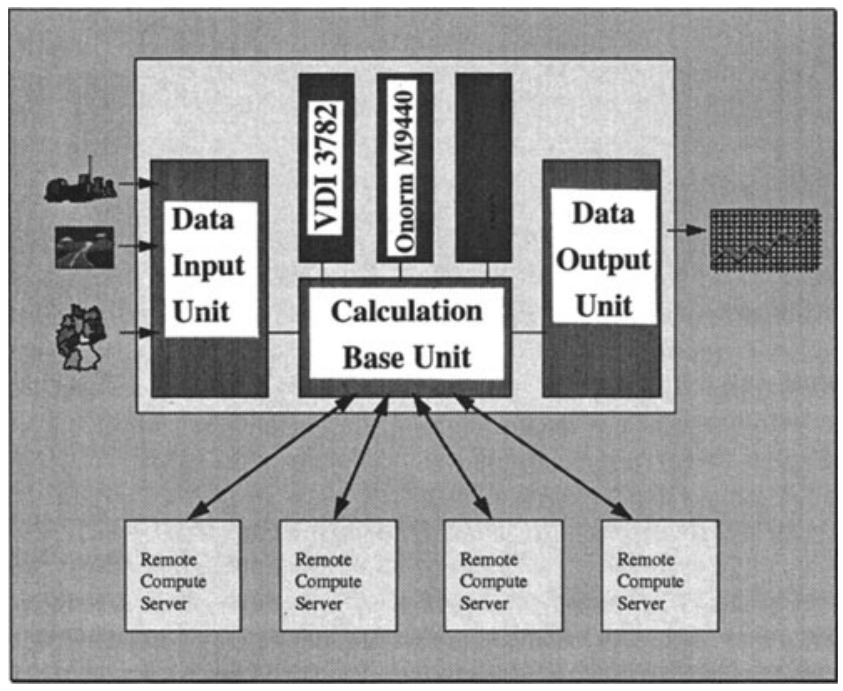

Figure 1 Architecture of our solution for simulation and visualisation of environmental information with job distribution. 
Graphical user interface and input of common data is done by the input unit. Preparation of simulated data for graphical and tabular output is task of the output unit. Due to our modular architecture the specific simulation models are optional, depending on individual needs. These optional units are built by inherited classes which usually exist of additional user interface components as well as the algorithms for simulation. Further input information needed for special air pollution dispersion models are handled by itself. We organized them as dynamic link libraries, a sample for a special approved austrian standard is given in chapter 3. The modular structure guarantees flexibility for adding different kinds of air pollution dispersion model (i.e. OENORM 9440, VDI 3782, Dutch model,..) and increases the reuse of the complex and powerful architecture. With respect to the compatibility of the different dispersion models we defined a generic interface between calculation base unit and the optional calculation modules. The modules are represented as object which are derived from the virtual base class and embedded into a dynamic link library. All the specific dialog boxes and necessary input data are handled by the themself. Therefore this architecture allows to adopt future standards very easily.

\subsection{Input Unit}

We handle different types of input information which can be entered by the user in different ways. These various forms of information build the basis for further calculation steps. The following list gives an overview of the basic input parameters required to characterize individual emission situations:

\section{Information about sources}

- position and dimensions (height, diameter) of the source

- list of emitted substances (i.e. SO2, CO, NO, NO2, $\mathrm{H} 2 \mathrm{~S}, .$. )

- mass flow of the emitted substances

- exhaust volume rate

- exhaust temperature at source height

\section{Information about receptor points}

- coordinates of the receptor point

All the emission sources defined by the user for a special situation contain flags for individual controlling activity during simulation, therefore various conditions and views can be evaluated. For configuring this variety of information we support different mechanisms:

- Geographical background information: Input of information from external data files is supported by special interfaces for importing different fileformats (BMP, DXF, Bitmaps,..). This background information enables an improved graphical representation and allows easier navigation for the user.

- Definition of sources and receptor points: Predefined symbols enable the graphical definition of an environmental situation in the Cartesian as well as the Gauß-Krüger coordinate system. The Cartesian coordinates are relative to a reference point, which is often simultaneously the zero point. The Gauß-Krüger coordinates relate every point of the earth to a definite point with respect to a reference meridian. If the Gauß-Krüger coordinates of all sources and receptor points are known, they could be entered simply. 
Source points must be configured additionally with lists of emitted substances and corresponding parameters.

- Individual drawings: A set of simple tools (line, box, circle,...) allows to draw individual information or critical areas with a graphical method.

- Standard parameters: Special dialogs allow to define various standard parameters for substances and air pollutants.

These tools allow the definition of individual emission environments. Therefore they establish the base for further simulation and the situation can be stored on external files for later reuse or new calculation.

\subsection{Calculation Unit}

The calculation unit receives it's information from the input unit and forwards results to the output unit for presentation. As the base object of our framework the mandatory base calculation module defines the interface for linking specific calculation modules. Additional models represented as dynamic link libraries can be bound. An example of an optional calculation model is given in chapter three. Furthermore the base module establishes the connection to the remote compute servers (see figure 1) which are responsible for job computation. Depending on available resources jobs are distributed to connected computers which are configured for remote processing. Within the calculation unit the specific models are responsible for partitioning and forwarding their jobs to the base unit which distributes them for calculation. Due to parallel calculation we get a speedup depending on the calculation model and on available resources on remote servers[Kum94] also. Figure 2 gives the benchmark results for the OENORM M9440 model which leads to a nearby linear speedup. The values for speedup depend on complexity and properties of the model as well as the available resources within the network.

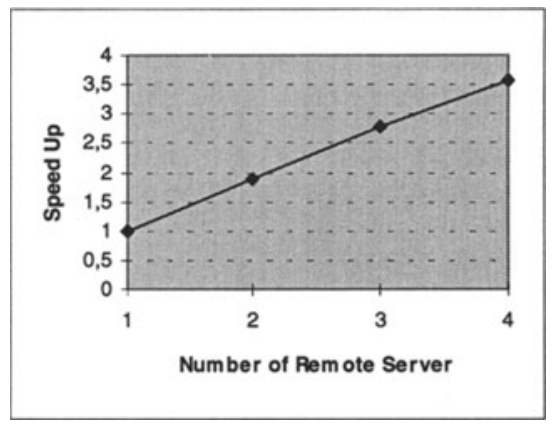

Figure 2 Speedup for parallel processing of the OENORM M9440 model.

The client / server communication between the base calculation unit and the connected remote servers is done by remote procedure calls[Cor91]. We have implemented mechanisms on the remote servers allowing performance and load evaluation. Compute jobs can be distributed to those machines, having free resources. Also the computer running the main application can take part in servicing calculation jobs. Therefore also local systems can execute our application and no minimum number of remote machines is required. The maximum number of computers for parallel calculation is limited by the increasing network traffic and the structure of the 
calculation model also. Time consuming jobs with a small amount of communication parameters are an indicator for gaining high speedups due to parallel processing with our architecture.

\subsection{Output Unit}

The output unit is a mandatory component which handles graphical and tabular representations as well as density charts. Different views to the results depending on configuration are available:

- Considering sources as stand alone - for viewing and comparing the effects with other sources

- Including all active sources within the emission area and calculating corresponding output

\section{CASE STUDY: INTEGRATION OF THE OENORM M9440 STANDARD}

Nowadays a wide range of calculation and simulation methods for dispersion models are known and standardized. Examples are the Austrian OENORM M9440, the German VDI 3782 or the Dutch model. We decided to implement the Austrian OENORM M9440 [OEN92] as the first air pollution dispersion model for our simulation framework. This standard is based on the Gaussian dispersion model[OEN92],[VDI92],[VDI91], which simulates the process of dilution and transport of emissions. Therefore, when applying this model, the emission data as well as the meteorological parameters must be known. The advantages of the Gaussian dispersion model compared to more complex models consist in the fact that the dispersion parameters used have been derived from dispersion investigation experiments. The Gaussian model contains approximations and simplifications which sometimes lead to systematic errors. In addition the inaccurate measurement of all input parameters can lead to errors in the calculated concentrations also. For these reasons, the Gaussian dispersion equation represents a dispersion model which has been validated empirically by dispersion experiments. Compared to more complex models, a relatively short computing time is needed.

Our implemented calculation module requests additional input from the user covering some basic meteorological parameters:

- Transport velocity of the wind

- Transport direction of the wind

- Dispersion categories

- Dispersion parameters

- Mixing layer height

One assumption for the correctness of this model is that these parameters are constant over the global emission for a specified period. Only this assumption guarantees the ability to use this calculation model. 


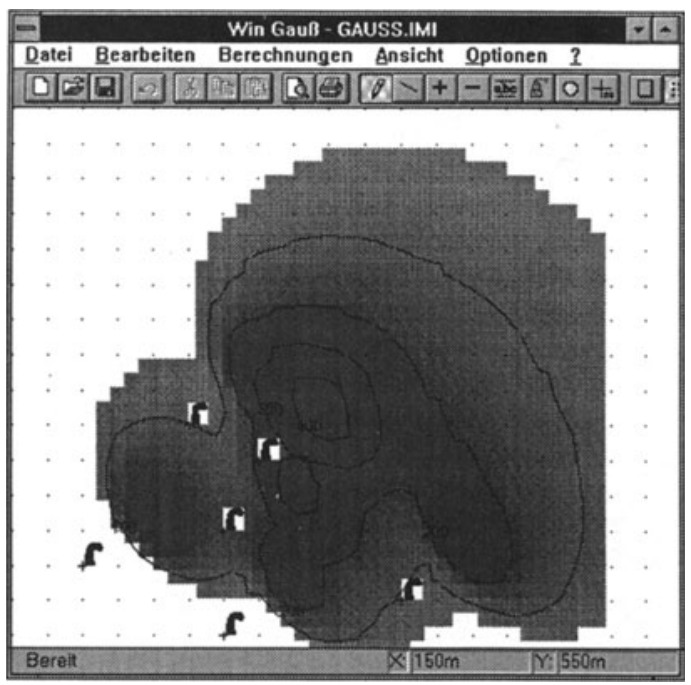

Figure 3 The graphical result for simulation of six sources of the air pollutant $\mathrm{SO}_{2}$ (transport direction 235 degree, air temperature $20^{\circ} \mathrm{C}$ ).

\section{CONCLUSION}

This article presents the architectural overview of our generic framework for simulation and visualisation of emission dispersion. Special simulation models can be linked into this framework. Therefore easy adaptation and extension is given by wrapping into dynamic link libraries. By partitioning the simulation models we can distribute jobs to connected computers for parallel computation and increasing performance.

Due to low resource requirement, our solution is suitable for desktop calculation in local and network environments. Fast and easy analyzing of emission situations with graphical data representation is the users advantage.

\section{REFERENCES}

[Cor91] Corbin, John R. The Art of distributed applications.

Programming techniques for remote procedure calls.

Springer Verlag, 1991

[Esc94] Eschelbeck G., Mühlbacher J.: A Framework of Classes for Distributed Controlling. Proc. EUROMICRO Conference 1994, pp. 240-246

IEEE Press, California, 1994

[Kum94] Kumar V., Grama A., Gupta A., Karypis G. Introduction to Parallel Computing. The Benjamin/Cummings Publishing Company.

California, 1994 
[OEN92] OENORM M9440. Ausbreitung von luftverunreinigenden Stoffen in der Atmosphäre. Berechnung von Immissionskonzentrationen und Ermittlung von Schornsteinhöhen.

[VDI92] VDI 3782 Part 1. Gaussian Dispersion Model for Air Quality Managment. Dispersion of Pollutants in the Atmosphere. October 1992

[VDI91] VDI 3782 Blatt 4. Ausbreitung von Geruchsstoffen in der Atmosphäre. Umweltmeteorologie. Mai 1991

\section{BIOGRAPHY}

Gerhard Eschelbeck is a graduate computer science engineer. In 1993 he joined the Research-Institute for Microprocessor-Applications at the University of Linz, Austria. His current research interests include networking and distributed computing. He is a member of the IEEE, the Computer Society, and the ACM and is an active participant in international computer science conferences.

Christian Hartl studied computer science at the Johannes Kepler University Linz, Austria. Main emphasis in the field of system programming, algorithm and data structures. Establishment of the diploma thesis at the Research Institute of Microprocessor Applications with the topic "Dynamic load distribution". Further interests in the field of Computer supported cooperative work (CSCW). 